\title{
Técnica de "mini-nuc" de Blumenthal: resultados de 454 casos
}

\author{
Mini-nuc Blumenthal technique: results of 454 cases
}

\author{
Mauro Waiswol ${ }^{1}$ \\ Ralph Cohen ${ }^{2}$ \\ Fabio Ejzenbaum ${ }^{3}$
}

\section{RESUMO}

Objetivo: Analisar retrospectivamente os resultados do tratamento cirúrgico de catarata pela técnica de "Mini-Nuc" de Blumenthal. Método: Foram, assim, submetidos à extração extracapsular de catarata pela referida técnica, realizada sempre pelo mesmo cirurgião (MW), 454 olhos de 284 pacientes ( 148 mulheres e 136 homens), em período de seis anos (fevereiro de 1994 a março de 2000). Resultados: Os resultados apontaram baixas taxas de complicações intra-operatórias (4,8\%), sendo que a da ruptura da cápsula posterior ocorreu em $3,7 \%$ dos casos. Correção da acuidade visual igual ou maior que 20/40 foi obtida em 89,1\% dos casos. Conclusão: A técnica mostrou-se, portanto, eficaz, com a vantagem de prescindir de instrumentação sofisticada e envolver baixos custos.

Descritores: Extração de catarata/métodos; Extração de catarata/economia; Câmara anterior/cirurgia; Acuidade visual

\section{INTRODUÇÃO}

Já são amplamente conhecidas as vantagens do uso da pequena incisão com até $3,2 \mathrm{~mm}$, no tratamento operatório da catarata. Estas técnicas manuais, como são referidas, vêm sendo realizadas como alternativa para a facoemulsificação que, por sua vez, já foi reconhecida internacionalmente como procedimento de escolha para a extração da catarata ${ }^{(1-3)}$.

Por outro lado, visando evitar os custos envolvidos com a facoemulsificação bem como reduzir as diferenças encontradas nos resultados da extração extracapsular (ECCE) programada tradicional e da cirurgia de pequena incisão, Blumenthal et al..$^{(4-8)}$ e Kansas e $\mathrm{Sax}^{(9)}$, entre outros cirurgiões, desenvolveram alternativas para a facoemulsificação que permitem, ainda, incisão média variando de 5 a $7 \mathrm{~mm}$, implantação de lente intra-ocular dentro do saco capsular e recuperação funcional rápida.

Blumenthal et al. ${ }^{(4-8)}$ propuseram o uso de um mantenedor de câmara anterior (MCA) na extração extracapsular manual programada de catarata, $o$ qual possibilita reposição imediata, espontânea e concomitante de fluido eventualmente perdido, controlando, deste modo, a pressão intra-ocular intra-operatória. O "Mini-Nuc" será obtido através da separação do epinúcleo com o núcleo cristaliniano através da hidrodelineação.

A técnica "Mini-Nuc", como é hoje conhecida, descrita por Blumenthal, combina, portanto, os benefícios da incisão média sem sutura com a extração extracapsular manual da catarata, utilizando o MCA que, por meio de pressão hidrostática interna somada a leve pressão externa, exterioriza o mini-núcleo através da incisão tunelizada. Neste procedimento, o MCA permite fluxo constante de irrigação, promovendo pressão intra-ocular positiva durante todo o ato operatório.
Pós-graduando em Nível de Doutorado na Faculdade de Medicina da Universidade de São Paulo, Chefe da Seção de Catarata do Departamento de Oftalmologia da Santa Casa de São Paulo.

(a) Adjunto da Faculdade de Ciências Médicas da Santa Casa de São Paulo.

${ }^{3}$ Médico Estagiário do Departamento de Oftalmologia da Santa Casa de São Paulo.

Endereço para correspondência: Av. Angélica, 916 - cj.903 - São Paulo (SP) CEP 01228-000. E-mail: waiswol@poboxes.com 
O presente estudo analisa retrospectivamente os resultados do tratamento de 454 olhos operados pelo mesmo cirurgião (MW), empregando a técnica de "Mini- Nuc" de Blumenthal ${ }^{(4-8)}$.

\section{MÉTODOS}

\section{Casuística}

Foram selecionados para este estudo 284 pacientes atendidos e tratados no Departamento de Oftalmologia da Santa Casa de São Paulo ou em clínica particular, no período compreendido entre fevereiro de 1994 e março de 2000, os quais apresentavam apenas catarata, sem qualquer outra patologia ocular. Este grupo de pacientes compunha-se de 148 mulheres e 136 homens, com idades oscilando entre 39 e 87 anos, totalizando 454 olhos operados sempre pelo mesmo cirurgião.

Todos os pacientes foram submetidos a exame oftalmológico completo, incluindo refração, acuidade visual corrigida (Snellen), ceratometria, biomicroscopia com e sem dilatação da pupila, tonometria de aplanação, oftalmoscopia e biometria.

\section{Técnica operatória}

Para o ato operatório, utilizou-se anestesia geral em 11 casos (2,5\%); anestesia local pela técnica peribulbar com lidocaína a $2 \%$ em 89 casos $(19,6 \%)$ e anestesia tópica com cloridrato de proximetacaína mais injeção intracamerular de cloridrato de lidocaína a 2\% sem conservantes em 354 casos (77,9\%).

Em todos os casos, realizava-se peritomia conjuntival limbar superior, com base no fórnix. Após diatermia da esclera exposta, procedia-se a uma incisão escleral curvilínea ("frown incision") de $5 \mathrm{~mm}$ a $7 \mathrm{~mm}$ de extensão, que deveria alcançar a metade de sua espessura e situar-se a 1,5 mm do limbo (transição córneo-escleral).

Para a realização do túnel escleral, utilizou-se bisturi angulado do tipo crescente, com corte voltado para cima de $2,25 \mathrm{~mm}$ de largura. A dissecção avançava anteriormente, até atingir distância de $1,5 \mathrm{~mm}$ da córnea clara, com a parte interna da incisão sendo $20 \%$ maior do que a parte externa escleral. Utilizava-se, então, cerátomo de 3,2 $\mathrm{mm}$ de largura, que era inserido através do túnel, até penetrar a câmara anterior. O movimento do cerátomo para a direita e para a esquerda delimitava a extensão da incisão.

Na seqüência, foram feitas duas incisões corneanas autoselantes: a primeira, de serviço, de $1 \mathrm{~mm}$ de extensão, sempre no meridiano de $10 \mathrm{~h} 00$ e a segunda, de $1 \mathrm{~mm}$ de extensão, no quadrante inferior, por onde era, então, instalado o MCA, que era conectado, através de um tubo de silicone, a um frasco de solução salina balanceada (BSS). A altura do frasco podia ser alterada durante o ato operatório, de modo a permitir controle da pressão intra-ocular desejável nas diferentes etapas da operação.
A capsulorrexe foi feita com o uso de um cistítimo, que era introduzido através da incisão de serviço. Durante a capsulorrexe, o fluxo contínuo de fluido pelo MCA aprofundava a câmara anterior, aplanando a cápsula anterior do cristalino, tornando esta manobra mais segura.

Em seguida, foram realizadas a hidrodissecção e a hidrodelineação. A cânula hidrodissectora era introduzida entre o epinúcleo e o núcleo, até atingir a porção posterior do núcleo duro, que era deslocado para a câmara anterior através da capsulorrexe. Com o mini-núcleo já posicionado na câmara anterior, era então possível determinar o tamanho necessário para a incisão escleral.

Um deslizador ("Sheets glide") era, então, introduzido na câmara anterior através do túnel escleral e dirigido abaixo do mini-núcleo. A pressão hidrostática interna do MCA e ligeira pressão externa eram utilizadas, às $12 \mathrm{~h} 00$, para exteriorizar o mini-núcleo; a mesma manobra era aplicada ao epinúcleo.

A aspiração da córtex, feita com uma cânula com $0,4 \mathrm{~mm}$ de abertura, introduzida pela incisão de serviço. A lente era, então, implantada no saco capsular, que se mantinha inflado sob ação do MCA.

Embora a incisão fosse auto-selante, em alguns casos foi necessária a sua ampliação além dos $5 \mathrm{~mm}$, alcançando até 7 $\mathrm{mm}$ dependendo da extensão do núcleo, ocasiões em que se recomendou sutura com dois pontos separados de mononylon 10-0, o que representou pequena modificação à técnica original. Foram implantadas lentes intra-oculares, de polimetilmetacrilato rígidas, de peça única com zona óptica que variaram de $5 \mathrm{~mm}$ a $6 \mathrm{~mm}$ de diâmetro.

Em seguida, era removido o MCA, e o estroma corneano tanto desta região quanto da incisão de serviço foi hidratada. A conjuntiva foi recolocada com o uso da diatermia e realizada injeção subconjuntival de gentamicina e dexametasona.

O tratamento pós-operatório incluía instilação de tobramicina quatro vezes ao dia por duas semanas e de dexametasona quatro vezes ao dia por quatro semanas.

No seguimento e avaliação pós-operatórios, analisou-se a evolução da acuidade visual e presença de astigmatismo corneal; realizavam-se, ainda, fundoscopia, tonometria e biomicroscopia. A refração final foi prescrita aos 60 dias de pósoperatório, período que representou o tempo de seguimento mínimo destes casos.

\section{Avaliação dos resultados}

Para a avaliação dos resultados, considerou-se o registro das complicações intra-operatórias realizado imediatamente após a cirurgia, bem como a melhor correção da acuidade visual no $60^{\circ}$ dia de pós-operatório.

\section{RESULTADOS}

Incisões auto-selantes foram possíveis em 318 dos olhos operados $(70,0 \%)$, enquanto 136 olhos $(30,0 \%)$ necessitaram de suturas adicionais. 
Capsulorrexe circular contínua foi obtida em 411 olhos (90,5\%).

As complicações intra-operatórias registradas incluíram 11 casos de ruptura da cápsula posterior sem perda vítrea $(2,4 \%)$; seis casos de ruptura da cápsula posterior com perda vítrea $(1,3 \%)$; três casos de ruptura parcial da zônula $(0,7 \%)$ e dois casos de luxação do núcleo para a câmara vítrea $(0,4 \%)$ (Gráfico 1 - A, B). Ruptura da cápsula posterior sem ou com perda vítrea somaram, assim, $3,7 \%$ dos casos.

O gráfico 2 evidencia a melhor correção da acuidade visual no $60^{\circ}$ dia de pós-operatório, mostrando que, em 404 casos $(89,1 \%)$, esta foi igual ou maior que 20/40.

$\mathrm{O}$ astigmatismo médio induzido, avaliado no $60^{\circ}$ dia de pós-operatório, foi de 0,87 dioptrias.

Os casos em que a acuidade visual se mostrou menor que 20/40 apresentavam associação com degeneração senil macu-

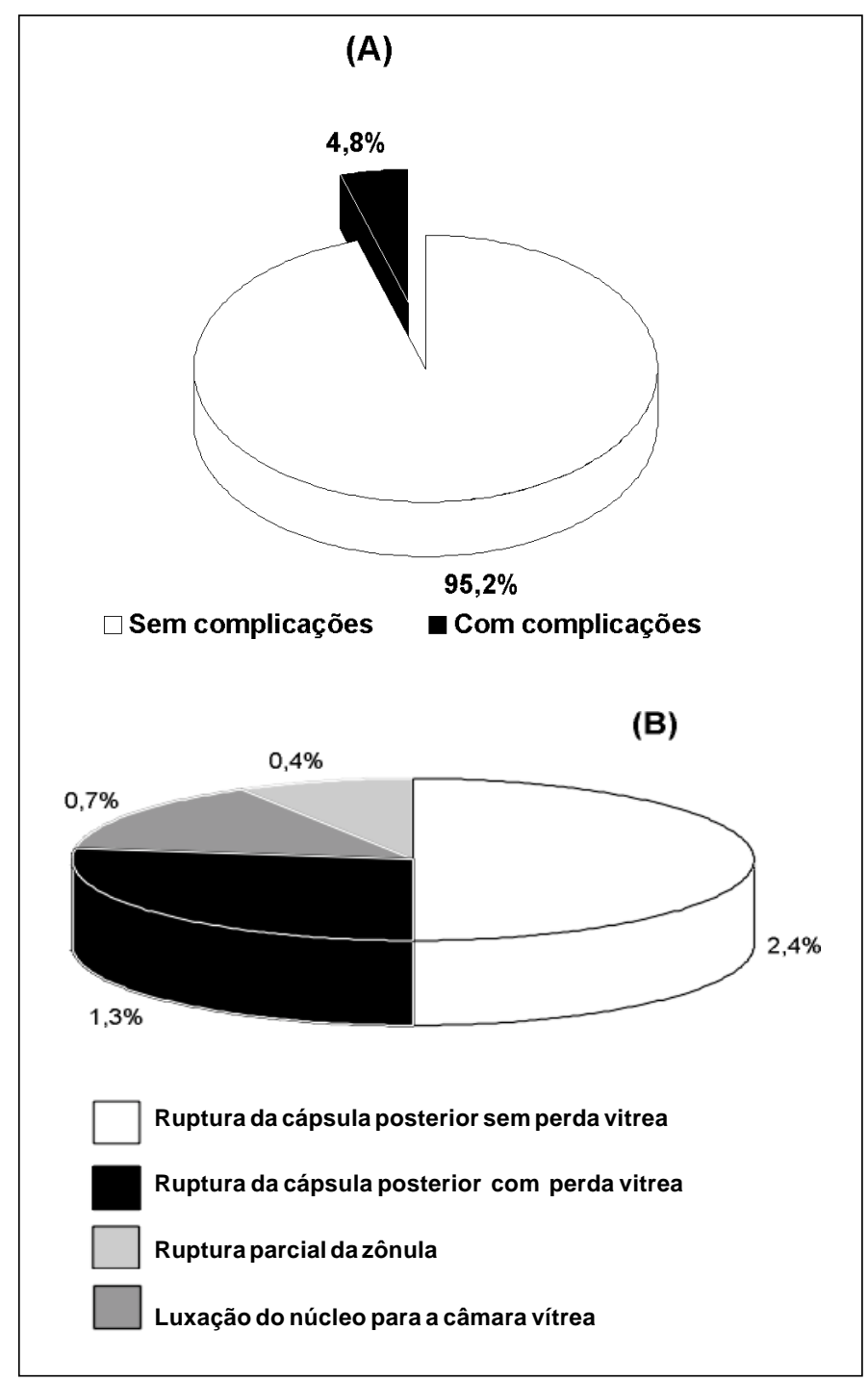

Gráfico 1 - Complicações intra-operatórias registradas para 454 olhos com catarata operados pela técnica de "Mini-Nuc" de Blumenthal (Santa Casa de São Paulo, 1994-2000)

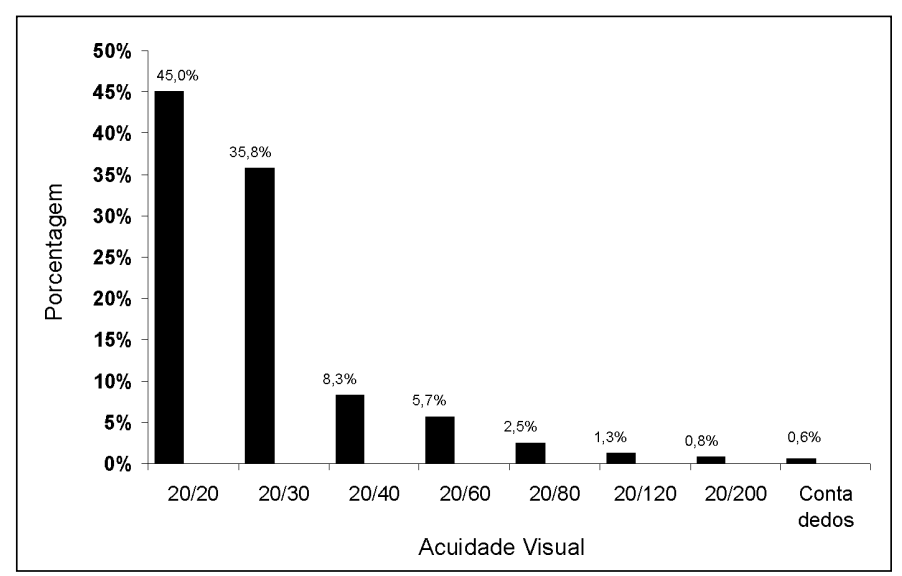

Gráfico 2 - Melhor correcão da acuidade visual registrada no $60^{\circ}$. dia de pós-operatório para 454 olhos com catarata operados pela técnica de "Mini-Nuc" de Blumenthal (Santa Casa de São Paulo, 1994-2000)

lar, edema macular cistóide, maculopatia miópica, glaucoma avançado e/ou buraco macular idiopático.

\section{DISCUSSÃO}

Todas as técnicas operatórias propostas para tratamento da catarata visam sobretudo o sucesso na reabilitação visual. As técnicas de pequena incisão auxiliam neste objetivo, além de diminuir $\mathrm{o}$ astigmatismo induzido operatoriamente e propiciar recuperação pós-operatória mais rápida ${ }^{(21)}$.

Desde que Kelman ${ }^{(10)}$ introduziu a facoemulsificação, a tecnologia de ultra-som progrediu muito rapidamente, fazendo desta técnica a de primeira escolha entre os mais exigentes cirurgiões. No entanto, trata-se de técnica que requer equipamentos caros e que exige curva de aprendizado relativamente longa, desvantagens essas que podem ser supridas com as técnicas manuais de média incisão, com as quais são obtidos benefícios terapêuticos semelhantes.

Assim, quando se deseja aliar custos baixos a bons resultados, dispõe-se da técnica "Mini-Nuc" como excelente alternativa ${ }^{(11-14)}$, a qual combina os benefícios da média incisão auto-selante com a extração manual extracapsular da catarata, propondo a utilização de um MAC que promove fluxo contínuo de BSS e garante pressão intra-ocular positiva durante toda a operação.

A grande vantagem desta técnica de média incisão está no fato de não requerer uso de instrumentação sofisticada, fazendo uso de cânulas força hidráulica, simplificando as manobras operatórias necessárias à remoção do núcleo e epinúcleo. $\mathrm{O}$ fluxo constante do MCA mantém inflado o saco capsular, reduzindo os riscos de ruptura da cápsula posterior, durante a aspiração dos restos corticais e durante o implante da lente intra-ocular.

Em nossa série, as incisões auto-selantes foram possíveis em 318 dos olhos operados (70,0\%). Kimura et al. ${ }^{(15)}$ relataram incisões auto-selantes em $88,2 \%$ de 51 olhos operados com a 
técnica de extração extracapsular com incisão sem sutura proposta por Fry ${ }^{(16)}$.

Além do grau de reabilitação visual, o registro de eventuais complicações intra-operatórias, especialmente as relativas à ruptura da cápsula posterior, constitui meio eficaz para a avaliação das diferentes técnicas propostas para a correção da catarata.

Registramos 4,8\% de ocorrências de complicações intra-operatórias com o emprego da técnica de "Mini-Nuc "(Gráfico 1).

Duch Mestres et al. ${ }^{(17)}$ compararam a ocorrência de complicações intra-operatórias em 444 olhos operados com ECCE e 123, com a técnica de nucleofragmentação manual. No intraoperatório de ECCE, registraram ocorrência de complicações na ordem de $39,2 \%$, enquanto os casos operados com nucleofragmentação manual tiveram taxa de complicações de 27,4\%. Não foi estatisticamente significativa a diferença das taxas registradas.

Saccá et al. ${ }^{(18)}$ analisaram os resultados de 200 casos operados pela técnica de captura de núcleo com pequena incisão, relatando complicações intra-operatórias em 65 casos (32,5\%). Ruptura da cápsula sem perda vítrea ocorreu em 1,5\% dos casos; com perda vítrea, em 1,5\%; prolapso transiente da íris, em 22,0\%; e prolapso permanente da íris em 1,5\%.

Em nosso meio, nos cem primeiros casos tratados com facoemulsificação por José e Felício da Costa ${ }^{(19)}$, houve taxa de ruptura da cápsula posterior de $14,0 \%$, sendo que $88,0 \%$ destes casos apresentaram acuidade visual de 20/40 após quatro semanas da cirurgia. Já nos cem primeiros casos submetidos à mesma técnica por Araújo et al. ${ }^{(20)}, 8,7 \%$ dos casos apresentaram ruptura da câmara posterior e $5,4 \%$, perda vítrea; um caso apresentou luxação de núcleo no vítreo. A acuidade visual final foi igual ou maior que 20/40 em $90 \%$ dos casos.

Ventura et al. ${ }^{(20)}$, por sua vez, relataram os resultados de 100 olhos de 84 pacientes tratados com a técnica de facoemulsificação com incisão em córnea clara às $11 \mathrm{~h} 00$ e implante de lente intra-ocular de silicone. Estes autores referiram não ter encontrado quaisquer complicações transoperatórias e ter obtido acuidade visual final de $20 / 40$ a 20/20 em $80,5 \%$ dos casos.

Já nos cem primeiros casos relatados por Teles et al. ${ }^{(21)}$, a complicação intra-operatória mais freqüente em operação que usou a técnica da facoemulsificação foi a perda vítrea por ruptura de cápsula posterior $(11, \%)$, seguida por trauma da íris $(3,5 \%)$. Utilizando a mesma técnica em 88 casos consecutivos, Matuda et al. ${ }^{(22)}$ registraram taxa de ruptura de cápsula posterior de $21,5 \%$ e de perda vítrea de $12,5 \%$; a acuidade visual final foi igual ou maior que $20 / 40 \mathrm{em} 83,9 \%$ dos casos.

Grande parte destes autores ${ }^{(19,21-22)}$ conclui pela eficácia e segurança da facoemulsificação, desde que adotada estratégia adequada para este aprendizado e desde que realizada por cirurgiões experientes e familiarizados com a técnica.

À luz de nossos achados, concordamos com Chawla et al. ${ }^{(14)}$ no sentido de que há um rol de vantagens na utilização de um MAC para hidroexpressão do núcleo cristaliniano, através da média incisão, sem uso de agentes viscoelásticos ou de facoemulsificação, tais sejam:
- Maior segurança na técnica operatória no segmento anterior que se fundamente em incisão auto-selante pela criação e manutenção de espaço na câmara anterior;

- Possibilidade de manutenção da câmara anterior constantemente profunda, o que reduz a tensão dentro da cápsula da lente anterior, permitindo uma capsulorrexe mais fácil e mais segura;

- Manutenção da câmara anterior constantemente profunda, o que ajuda a manter dilatação pupilar;

- O uso do MAC auxilia no aprendizado da conversão de ECCE para facoemulsificação, uma vez que a aspiração de córtex residual com emprego de extrator cortical por meio de paracentese simples ocorre dentro de um saco capsular amplamente expandido;

- Na facoemulsificação, o MAC mantém a câmara anterior profunda em todos os momentos, especialmente na ocasião da retirada da ponteira do facoemulsificador do olho, permitindo que a sua reinserção seja mais fácil e menos traumática para a íris;

- Uma lente intra-ocular pode ser inserida com segurança no saco capsular amplamente expandido sem o uso de agentes viscoelásticos;

- Por fim, a tendência de fluxo constante pode reduzir o risco de contaminação bacteriana intra-ocular.

A técnica "Mini-Nuc" oferece, portanto, rápida reabilitação visual, pequeno astigmatismo induzido, baixa incidência de ruptura capsular e menor inflamação pós-operatória ${ }^{(14)}$, somando-se a isso a não necessidade de instrumentos sofisticados nem de agentes viscoelásticos; na maioria dos casos, o uso de fios de sutura também é dispensável.

\section{CONCLUSÃO}

As pequenas taxas de complicações intra-operatórias registradas em 454 olhos operados com a técnica de "MiniNuc" de Blumenthal fazem concluir pela sua eficácia, particularmente se considerando o tempo curto de recuperação pósoperatória e a necessidade de instrumentação mínima, o que a torna especialmente atraente para os países em desenvolvimento, vistos os baixo custos em que implica.

\section{ABSTRACT}

Purpose: In this retrospective study the results of surgical management of cataract by the Mini-Nuc Blumenthal technique were analyzed. Methods: ECCE with Mini-Nuc Blumenthal technique was carried out always by the same surgeon (M.W.) on 454 eyes of 284 patients ( 148 women and 136 men) during a period of six years (from February 1994 to March 2000). Results: Low rates (4.8\%) of transoperative complications were recorded; posterior capsule rupture occurred in $3.7 \%$ of the cases. Visual acuity equal to or higher than $20 / 40$ 
was observed in $89.1 \%$ of the cases. Conclusion: The technique was efficient for cataract surgery with the advantage of dispensing with sophisticated instrumentation and involving low costs.

Keywords: Cataract extraction/methods; Cataract extraction/ economy; Anterior chamber/surgery; Visual acuity

\section{REFERÊNCIAS}

1. Obstbaum SA. Phacoemulsification: the favored surgical technique (editorial). J Cataract Refract Surg 1991;17:267.

2. Gimbel HV. Divide and conquer nucleofractis phacoemulsification: development and variations. [commented on J Cataract Refract Surg 1991;17:267]. J Cataract Refract Surg 1991;17:281-91.

3. Nishi O, Nishi K, Sakka Y. Endocapsular cataract surgery following small circular capsulorhexis. Eur J Implant Refract Surg 1990;2:33-6.

4. Blumenthal M, Assia EI, Chen V, Avni I. Using an anterior chamber maintainer to control intraocular pressure during phacoemulsification. J Cataract Refract Surg 1994;20:93-6.

5. Blumenthal M, Assia E, Schochot Y. Lens anatomical principles and their technical implications in cataract surgery. Part I: The lens capsule. J Cataract Refract Surg 1991;17:205-10.

6. Blumenthal M, Assia E, Neuman D. Lens anatomical principles and their technical implications in cataract surgery. Part II: The lens nucleus. J Cataract Refract Surg 1991;17:211-7.

7. Blumenthal M, Moisseiev J. Anterior chamber maintainer for extracapsular cataract extraction and intra-ocular lens implantation. J Cataract Refract Surg 1987;13:204-6.

8. Blumenthal M, Ashkenazi I, Assia E, Cahane M. Small incision manual extracapsular cataract extraction using selective hydrodissection. [commented on Ophthalmic Surg 1993;24:135]. Ophthalmic Surg 1992;23:699-701.

9. Kansas PG, Sax R. Small incision cataract extraction and implantation surgery using a manual phacofragmentation technique. J Cataract Refract Surg 1988;14:328-30.

10. Kelman CD. Phaco-emulsification and aspiration: a new technique of cataract removal. A preliminary report. Am J Ophthalmol 1967;64:23-35.

11. Welsh RC. Avoid subtle pitfalls in Blumenthal's ECCE with mini-nucleus hydroexpression: attention to detail makes this sutureless, viscoelastic-free technique perfect for cost-constrained surgery. Ocular Surg News 1994; June:27-8.

12. Welsh RC. Mini-nucleus external "chipping" method allows low-cost, sutureless, $4.5 \mathrm{~mm}$ ECCE without use of viscoelastics. Ocular Surg News 1994; September:44-5.

13. Mandle M. Mini-nuc technique for ECCE reduces tunnel size. Ocular Surg News 1996; March:9.

14. Chawla HB, Adams AD. Use of the anterior chamber maintainer in anterior segment surgery. J Cataract Refract Surg 1996;22:172-7.

15. Kimura H, Kuroda S, Mizoguchi N, Terauchi H, Matsumura M, Nagata M. Extracapsular cataract extraction with a sutureless incision for dense cataracts. J Cataract Refract Surg 1999;25:1275-9.

16. Fry LL. The phacosandwich technique. In: Rozakis GW, editor Cataract surgery: alternative small incision techniques. Thorofare, Slack, 1990. p.71-110.

17. Duch Mestres F, Matheu A, Torres F, Lillo J, Castillo M. Intraoperative complications of planned extracapsular cataract extraction versus manual nucleofragmentation. [commented on J Cataract Refract Surg 1997;23:148-9]. J Cataract Refract Surg 1996;22:1113-5.

18. Saccá SC, Patrone G, Macrí A, Rolando M. Small incision nucleus capture: results of 200 cases. J Cataract Refract Surg 1999;25:969-74.

19. José LSG, Felício da Costa EG. Facoemulsificação: o aprendizado e os primeiros 100 casos. [resumo 86] Arq Bras Oftalmol 1997;60:399.

20. Araújo MEX, Chou AC, Silva CR, Oliveira LB. Resultados dos 100 primeiros casos de facoemulsificação realizados no Hospital do Servidor Público Estadual de S. Paulo. [resumo 6]. Arq Bras Oftalmol 1998;61:404.

21. Ventura M, Melo C, Arruda Jr. R, Ventura LO. Eficácia da facoemulsificação com incisão em córnea clara às 11h00: resultados de 100 olhos. [resumo 23]. Arq Bras Oftalmol 1998;61:434.

22. Matuda E, Amaral M, Oliveira DF, Castro RS, Arieta CEL. Resultados visuais e complicações na transição para a facoemulsificação. [resumo 73]. Arq Bras Oftalmol 1996;59:377.

\title{
XXXI CONGRESSO BRASILEIRO DE OFTALMOLOGIA
}

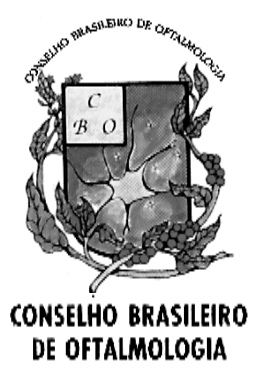

\author{
5 A 9 DE SETEMBRO DE 2001
}

\author{
CENTRO DE CONVENÇÕES IMIGRANTES \\ SÃO PAULO • SP
}

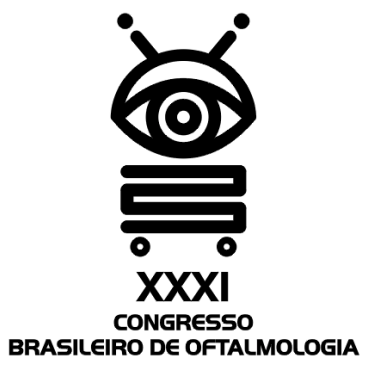

- Fórum de Residentes em Oftalmologia

- Conferência CBO - "2001 - A Odisséia do Glaucoma"

- Tema Oficial - "Senilidade Ocular"

- Sessão de Mobilidade Profissional

- Sessão de Abertura do Congresso - 5 de Setembro 